\title{
A Tree Formulation for Signaling Games with Noise
}

\author{
Xeni Dassiou, Dionysius Glycopantis \\ Department of Economics, City University London, London, UK \\ Email: x.dassiou@city.ac.uk
}

Received 26 June 2014; revised 29 July 2014; accepted 11 August 2014

Copyright (C) 2014 by authors and Scientific Research Publishing Inc.

This work is licensed under the Creative Commons Attribution International License (CC BY). http://creativecommons.org/licenses/by/4.0/

C) $\underset{\mathrm{EY}}{0}$ Open Access

\section{Abstract}

The paper provides an analysis of a sender-receiver sequential signaling game. The private information of the sender is transmitted with noise by a Machine, i.e. does not always correctly reflect the state of nature. Hence, a truthful revelation by the sender of his information does not necessarily imply that the signal he sends is correct. Also, the receiver can take a correct action even if the sender transmits an incorrect signal. The payoffs of the two players depend on their combined actions. Perfect Bayesian Equilibria which can result from different degrees of noise is analysed. The Bayesian updating of probabilities is explained. The fixed point theorem which makes the connection with the idea of rational expectations in economics is calculated. Given a number of equilibria, we comment on the most credible one on the basis of the implied payoffs for both players. The equilibrium signals are an example of the formation of a language convention discussed by D. Lewis.

\section{Keywords}

Signals and Non-Cooperative Signaling Games, Noise, States of Nature, A Machine, Imperfect Information Sets, Actions, Language Convention, Nash Equilibrium, Beliefs Updating, Perfect Bayesian Equilibrium, Fixed Point, Self-Fulfilling Prophesies, Rational Expectations Equilibrium

\section{Introduction}

In this paper we use the Lewis [1] formulation of signaling and base our model on our earlier paper (Dassiou and Glycopantis [2]), where we analysed a signaling game using a decision tree formation. The earlier paper presents a number of Bayesian equilibria and explains which one is likely to prevail using as a criterion the expected payoffs that they entail for the two players. A distinction between a true signal by a sender and a correct action by a receiver is established in terms of leading to different payoffs. A correct action by the 
receiver of a signal alone is not sufficient for leading to an equilibrium that involves maximum payoffs for both players. Moreover there are equilibria that do not involve maximum payoffs for either player.

Signaling games lead to the formation of language through combinations of signals and actions. In this paper we use the same signaling game as in [2]. The helper, $(H)$, referred to also as he, stands behind a truck gesturing to the driver, $(D)$, referred to also as she, to help her steer the truck into a narrow parking space. We assume that Nature consists of the particular position of the truck and of the parking space and that there are two such alternatives, each with probability $\frac{1}{2}$. Unlike the earlier paper, the sender is no longer fully aware of the state of nature, but rather receives a message with a noise regarding this. While both players are aware of the probabilities attached to the choices of nature, $H$ alone has private information (in the form of this message) and he signals to $D$ to direct her how to park her car.

Lewis describes the signaling behaviour and the action that follows as a "conventional regularity" of unwritten rules of parking gestures, based on experience to which all parties conform. In terms of highest payoffs there is a common interest objective of $H$ and $D$ to get the truck into the space. In this situation they both receive their optimal payoffs.

This is not always the case. A deviation from this rule, perhaps based on lack of trust, could lead to one or both of the two players getting inferior payoffs. In [2] there is a distinction between true or untrue signals sent by $H$ and correct or incorrect actions by the driver. The issue is to investigate whether a "correct" interpretation of the signals is possible, i.e. one which would lead to an optimal payoffs equilibrium position. In such a situation no one would wish to independently change his action if all the information was revealed and moreover the payoffs would be optimal.

In general, we can imagine that there are $n$ alternative states of nature, $T_{i}, \forall i=1,2, \cdots, n$, observed by the sender, $H$, who will send a signal concerning the private information (messages) he has received regarding the state of nature. The messages received by $H$ are not precise, but correct with a probability $1-q$. The sender compiles a set of alternative signals $s_{1}, s_{2}, \cdots, s_{j}, \cdots, s_{n}$ using a function $F_{H}:\left\{T_{i}\right\} \rightarrow\left\{s_{j}\right\}$. This is an encoding rule according to Blume [3]. The function $F_{H}$ translates (truthfully or not) the messages regarding the states of nature into $n$ communicated signals.

The receiver, $D$, then has to choose a response without knowing the state of nature. However he is aware of the prior probabilities of these states and the accuracy of the sender's private information. In the sequential game, $D$ translates these signals (decodes according to [3]). He chooses a best response $\rho_{k}$, from alternative actions $\rho_{1}, \rho_{2}, \cdots, \rho_{k}, \cdots, \rho_{n}$, to the signals. He applies Bayes' rule to form a posterior assessment that the signal comes from each state of nature by setting a decoding function $F_{D}:\left\{\rho_{k / s_{j}, q}\right\} \rightarrow\left\{T_{i}\right\}$. Rubinstein [4], notes the difficulty of formulating communication models into game theory models. Solutions in the latter are invariant to a change in the names of the actions that lead to these outcomes, i.e. alternative conventions leading to the same outcome.

A characteristic of the sender-signal-receiver-action sequential game paradigm is a plethora of equilibria. This raises the question of how to choose among such equilibria. Cho and Kreps [5] propose to eliminate some of these equilibria by branding them as "unintuitive". They restrict the out-of-equilbrium beliefs of the second party, D, i.e. interpretations that $D$ would have given to the signal that $H$ might have sent, but in equilibrium does not. Such beliefs might upset the given equilibrium. Cho and Kreps posit an intuitive explanation according to which the equilibrium introduces a self-reinforcing behaviour that is common knowledge among the players. By giving an emphasis to the equilibrium (outcome) payoffs any deviation from it is perceived as a conscious defection from the outcome. Therefore it needs to be justified on the basis of how it compares to that outcome. This emphasis carries on to our article in terms of choosing the most probable equilibrium outcome.

We assume that for each state of nature there is one action that has to be selected. To the best of our knowledge, the prevailing assumption in the literature is that if $D$ takes the "correct" action then maximum payoffs will be received by both $H$ and $D$ irrespective of whatever signal $H$ has sent. See, for example, [1], Pawlowitsch [6], Huttegger [7]. This is a game of common interest in which a resolution leads to optimal payoffs for both actors as noted by Skyrms [8].

This type of analysis is not complete on two counts. First, there is little discussion of the case where the action of the receiver may be appropriate (e.g. "correct") to the state of nature even if the signal sent is not. Second, 
there is no discussion of what happens to the payoffs of the two agents when this is the case. Lewis, and most recently philosophers like Stokke [9] make an attempt to discuss what constitutes "true" and "untrue" signals by a sender and responses in a signaling system. However there is no discussion of "truthfulness" in relation to "correctness".

Paper [2] departed from the assumption of a correct decision by the receiver leading to an optimal payoff for both. It distinguished between true signals (by $H$ ) and correct actions (by $D$ ). Unlike [1] and the majority of the subsequent literature, we noted that a correct action is not always the result of a truthful signal. $D$ may still correctly guess the state of nature although $H$ sends a "wrong" signal. A correct guess by $D$ means that she will receive the maximum payoff. $H$ will not if she has not truthfully revealed a signal that matches the true state of nature. Unlike [1], in our model a true signal is mutually rewarding if it leads to a correct decision by $D$ but a correctly guessed state of nature by $D$ is not necessarily so. We bind truthfulness (in the report of a signal) to a maximum payoff if accompanied by correctness, but the latter (in terms of the actions of $D$ ) is not solely the result of a truthful signal. $D$ may still correctly guess the state of nature although $H$ sends a "wrong" signal i.e. one that deviates from what he has seen. In this case $D$ receives the reward alone but $H$ does not. If $D$ ends up doing the incorrect thing then both get zero payoffs.

In our current paper of a signaling model with noise, $H$ receives a message that does not necessarily match with the actual true state of nature. Therefore, there is a de-coupling of the truthful revelation of the received information in the form of a signal dispatched by $H$ and whether this signal correctly reflects the state of nature. In other words, the delivery by $H$ to $D$ of a signal that does not match the true state of nature does not necessarily imply that the sender has been untruthful in revealing his signal. However, a signal that no longer reflects the true state of nature will imply a zero payoff for $H$. This is irrespective of whether or not it truthfully reflects the private information held by $H$ and irrespective of whether $D$ 's actions are correct or not.

\section{On a Simple Signaling Model with Noise}

We introduce the game theoretic analysis by considering a helper/driver example, as in our previous paper [2]. As in the previous model, there are two players, a helper $H$, (he), who tries to direct a driver $D$, (she), to park her car. "Nature" selects with probability $\frac{1}{2}$ the state to be either "left" $(L)$ or "right” $(R)$. It is now assumed that player $H$ picks up the correct state of nature with a noise indicated by probability $q$. We have again an imperfect information, non-cooperative signaling game, but this time it is rather more complicated, as the private information of $H$ regarding the state of nature in not precise. We are using again a game theoretic extensive form, dynamic decision tree formulation and the notation for pure and mixed strategies is obvious and that of the previous paper. The vectors of payoffs are given at the terminal nodes of the tree. The first element is the payoff of $H$ and the second that of $D$.

The rules for calculated payoffs are as follows. When $H$ ends up sending the "correct" signal and the correct action, i.e. the one corresponding to the actual state of the world, follows by $D$, then both players receive a payoff of 1. If $H$ communicates an incorrect signal and this leads to an incorrect action by $D$, then both players get 0 . If $H$ sends an incorrect signal and $D$ reacts choosing to play in the opposite direction then $D$ gets 1 for performing the correct action but $H$ ends up with zero.

In [2] we made the connection between the resolution of the signaling game without any noise and the ideas of rational expectations (self-fulfilling prophesies) from economic theory, which provide a formal interpretation of the results of our analysis. The expectations of the actors are self-fulfilling and they both receive their optimal payoffs. This connection between the concepts from the neighbouring disciplines of game theory and economics can also be established in the present case in which the existence of noise in the signals makes the game tree formulation more involved.

The structure of the game, the uncertainties introduced, the payoffs and the rationality of the players are common knowledge. Both players, $H$ and $D$, make rational decisions taking fully into account all the information which is common knowledge. The implications of their various strategies are clearly laid out. The players make rational predictions, (prophesies), of each others' actions and on this basis they act themselves. In the rational expectation equilibrium that results the predictions, that is the players' beliefs, are confirmed. The prophesies of the players are self-fulfilling. We give an explicit example of this below. 
We are concerned with the analysis of the implications of the signals sent by $H$ to $D$. The signal of nature and how it is read by the machine are both fixed with given probabilities and this is background information in the analysis. A machine picks up the signal from nature and transmits it to $H$. However, like all machines, it is not a $100 \%$ accurate. As the signal from nature goes to the machine it can be distorted. This means that if the machine shows on the screen "left" $L^{\prime}$, player $H$ will know that the real state is $L$ with probability $1-q$ and that with probability $q$ the real state is $R$. The situation is analogous if the machine shows on the screen "right" $\left(R^{\prime}\right)$. That is the machine could have distorted the original signal as it is transmitted on the screen. Player $H$ will know that the real state is $R$ with probability $1-q$, and $L$ with probability $q$. If the machine is only a bit faulty, we say that $q$ is the noise, due to the "trembling" hand of the machine. The smaller $q$ is, the more accurate is the transmission. If the machine is very faulty then the possibility of distortion, $q$, is large and we still call this "noise", as a technical term. Of course for $q=0$ we retrieve the previous model. ${ }^{1}$

The information sets $I_{1}$ and $I_{2}$ of $H$ capture the fact that he does not know for sure that what he sees at the screen is a true reflection of the state of nature. $I_{1}$ corresponds to seeing $L^{\prime}$ and $I_{2}$ to observing $R^{\prime}$. The information sets $I_{3}$ and $I_{4}$ belong to $D$ and capture the fact that she hears $l$ or $r$, respectively, but does not know what $H$ saw on the screen (e.g. $L^{\prime}$ or $R^{\prime}$ ). The two players can also play "left" or "right". In a pair of strategies for $H$ the first refers to $I_{1}$ and the second to $I_{2}$. In a pair of strategies for $D$ the first refers to $I_{3}$ and the second to $I_{4}$. As there is no risk of confusion, and in order to simplify matters, we use the same notation for the left and right moves from the nodes of information sets which belong to the same player. $H$ can play $l$ or $r$, and $D$ can play $l^{\prime}$ or $r^{\prime}$.

In a pair of strategies for $H$ the first refers to $I_{1}$ and the second to $I_{2}$. In a pair of strategies for $D$ the first refers to $I_{3}$ and the second to $I_{4}$.

We refer very briefly to two relevant equilibrium concepts in game theory. A Nash Equilibrium (NE) is a pair of strategies (actions) of the two players which are in terms of payoffs best replies to each other's.

A behavioural strategy assigns to the information sets of a player independent probability distributions to the actions available from those sets. The equilibrium concept of Perfect Bayesian Equilibrium (PBE) consists of a set of players' optimal behavioural strategies and consistent with these, a set of beliefs which attach a probability distribution to the nodes of each information set. Consistency requires that the decision from an information set is optimal given the particular player's beliefs about the nodes of this set and the strategies from all other sets, and that beliefs are formed from updating, using the available information. If the optimal play of the game enters an information set then updating of beliefs must be Bayesian. Otherwise appropriate beliefs are assigned arbitrarily to the nodes of the set. The PBE offers a dynamic interpretation of the solution noncooperative extensive form game. It makes use of the agents beliefs and it is a subset of NE.

The behavioural assumption is that every agent chooses his best strategy given the strategy of the other. That is, in effect, a reaction function is formed. If each player optimizes believing, (prophesyzing), a particular strategy for the other, and the outcome is that there is no reason for anybody to feel they have predicted wrongly, then we have an equilibrium which has been obtained rationally. The confirmation of the predictions takes places where the reaction functions intersect.

In terms of equilibria the distinction is between small $q<\frac{1}{2}$, and large $q>\frac{1}{2}$. In Figures 1-5, we have shown the optimal strategies of $H$ and $D$ with heavy lines. ${ }^{2}$ The calculations correspond to examples with $q$ small Figure 1 and Figure 2), large, (Figure 3 and Figure 4) and equal to $\frac{1}{2}$ (Figure 5). The expected payoffs, $E_{1}$ and $E_{2}$, of the two players are also given. It is straightforward to check that the strategies form a Nash Equilibrium (NE).

The calculations, through Bayesian updating, of the conditional probabilities, (beliefs), attached to the nodes of the information sets are based on the strategies and thus we obtain a PBE. The beliefs are shown on the nodes of the information sets. With respect to $I_{1}$ and $I_{2}$ they express the fixed probabilities with respect to which

\footnotetext{
${ }^{1}$ The beginning of the idea of $H$ receiving a false message from the machine regarding the state of nature was very briefly mentioned in [2], p. 5.

${ }^{2}$ We could interpret "Nature" and the "Machine” as two (passive) players, receiving, each, payoff zero, and playing the indicated mixed strategies.
} 
X. Dassiou, D. Glycopantis



Figure 1. $q$ small; $E_{1}=1-q, E_{2}=1-q$.

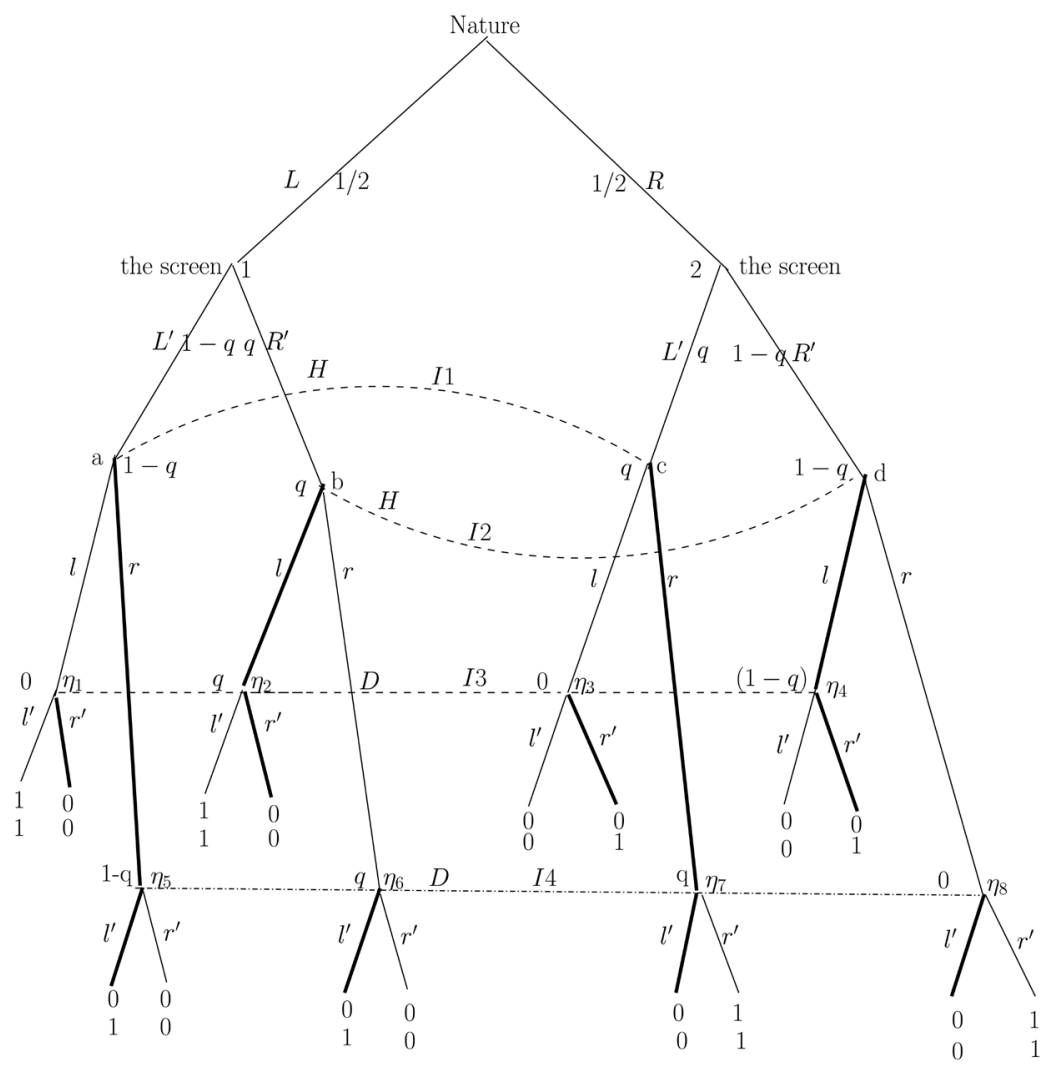

Figure 2. An alternative equilibrium with $q$ small; $E_{1}=0, E_{2}=1-q$.

1132 




Figure 3. An equilibrium with $q$ large; $E_{1}=0, E_{2}=q$.

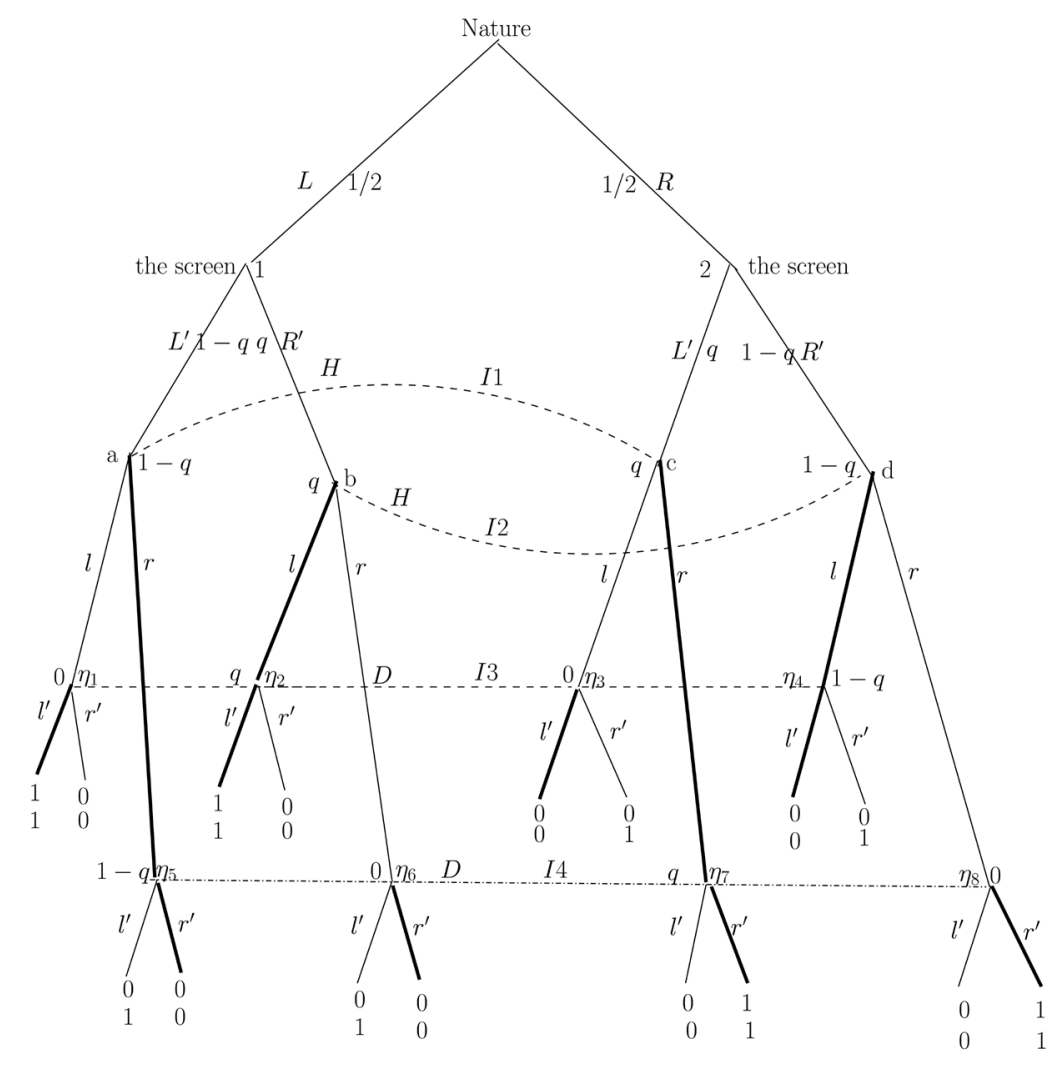

Figure 4. An alternative equilibrium with $q$ large; $E_{1}=q, E_{2}=q$. 


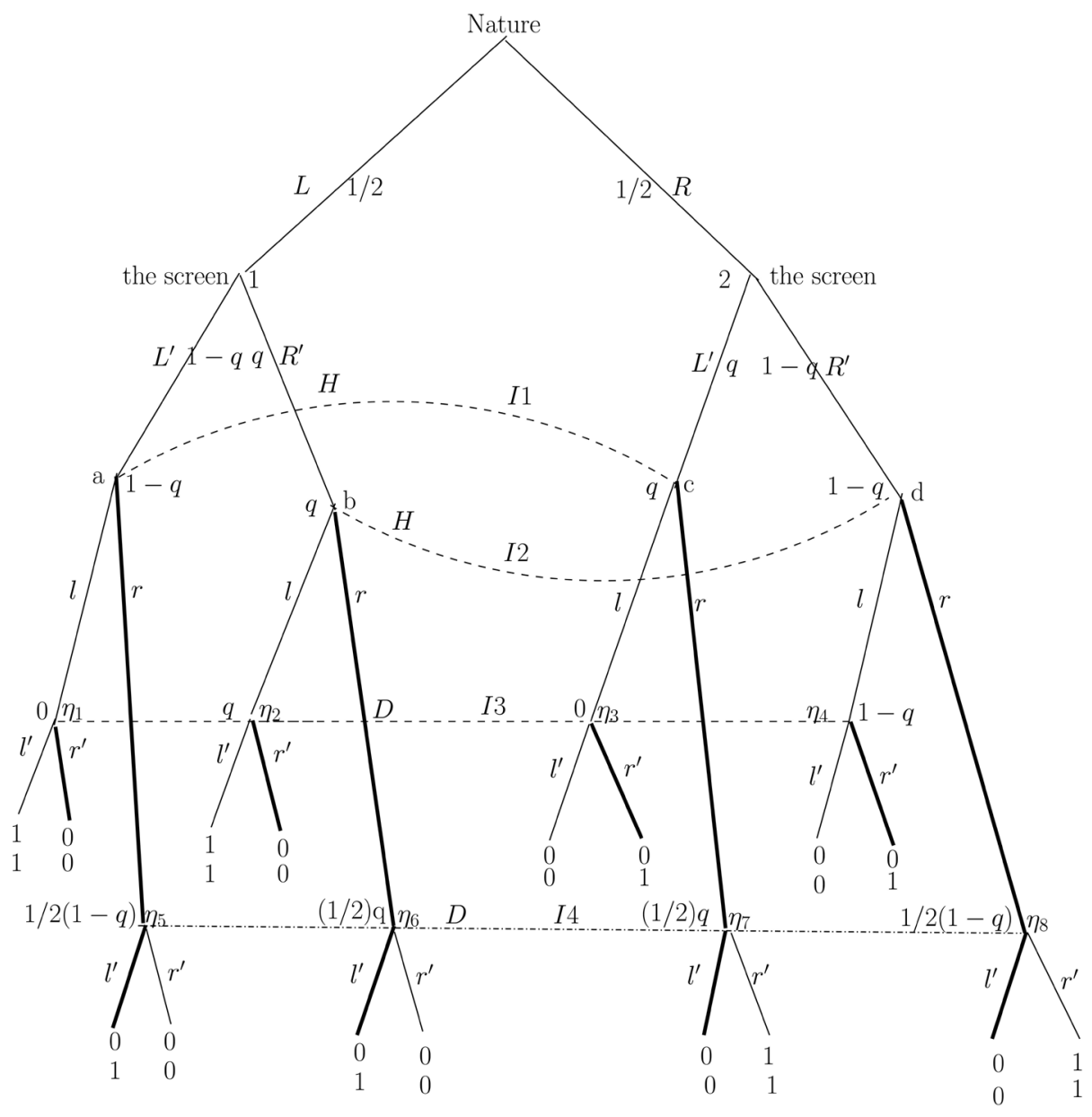

Figure 5. An equilibrium with $q=1 / 2 ; E_{1}=0, E_{2}=1 / 2$.

$H$ believes that a signal is true of false. ${ }^{3}$

In the end, we are interested in the players taking, from their information sets, decisions with probability one. In particular we want to analyse the case when $H$ will instruct $D$, with probability 1 , to turn "left" or "right" and $D$ will also play a pure strategy. Eventually we want to know which combination of pure strategies is most likely to prevail, i.e. whether the signal of $H$ will be truthful and if $D$ will believe it.

Case 1. $q$ is small. The optimal paths are shown in Figure 1. The information set $I_{3}$ contains the nodes, from left to right, $\eta_{1}, \cdots, \eta_{4}$, and we wish to calculate the beliefs attached to these by $D$. Using the Bayesian formula for updating beliefs, (see for example Glycopantis, Muir and Yannelis [10]), we can calculate these conditional probabilities. We know that $I_{3}$ is entered only if $H$ plays $l$. Hence

$$
\begin{aligned}
\operatorname{Pr}\left(\eta_{1} / l\right) & =\frac{\operatorname{Pr}\left(l / \eta_{1}\right) \times \operatorname{Pr}\left(\eta_{1}\right)}{\operatorname{Pr}\left(l / \eta_{1}\right) \times \operatorname{Pr}\left(\eta_{1}\right)+\operatorname{Pr}\left(l / \eta_{2}\right) \times \operatorname{Pr}\left(\eta_{2}\right)+\operatorname{Pr}\left(l / \eta_{3}\right) \times \operatorname{Pr}\left(\eta_{3}\right)+\operatorname{Pr}\left(l / \eta_{4}\right) \times \operatorname{Pr}\left(\eta_{4}\right)} \\
& =\frac{1 \times \frac{1}{2}(1-q)}{1 \times \frac{1}{2}(1-q)+1 \times 0+1 \times \frac{1}{2} \times q+1 \times 0}=1-q
\end{aligned}
$$

${ }^{3}$ In order to simplify the graphs, only in Figure 1 we have inserted explicitly that the actions of the players from the information sets can be mixed with probabilities as follows: $\left(l,\left(1-p_{1}\right) ; r, p_{1}\right)$ from $I 1,\left(l,\left(1-p_{2}\right) ; r, p_{2}\right)$ from $I 2,\left(l^{\prime},\left(1-z_{1}\right) ; r^{\prime}, z_{1}\right)$ from I3 and $\left(l^{\prime},\left(1-z_{2}\right) ; r^{\prime}, z_{2}\right)$ from $I 4$. Exactly the same notation is assumed in all the graphs. Also, only in Figure 1 we insert the folded up tree following the optimal decisions of $D$. Corresponding folded up trees can be obtained for all other figures displayed. 
Similarly we obtain the conditional probabilities $\operatorname{Pr}\left(\eta_{2} / l\right)=0, \quad \operatorname{Pr}\left(\eta_{3} / l\right)=q$ and $\operatorname{Pr}\left(\eta_{4} / l\right)=0$.

On the other hand $I_{2}$ is entered only if $H$ plays $r$. The information set $I_{2}$ contains the nodes, from left to right, $\eta_{5}, \cdots, \eta_{8}$. We now have

$$
\begin{aligned}
\operatorname{Pr}\left(\eta_{6} / r\right) & =\frac{\operatorname{Pr}\left(r / \eta_{6}\right) \times \operatorname{Pr}\left(\eta_{6}\right)}{\operatorname{Pr}\left(r / \eta_{5}\right) \times \operatorname{Pr}\left(\eta_{5}\right)+\operatorname{Pr}\left(r / \eta_{6}\right) \times \operatorname{Pr}\left(\eta_{6}\right)+\operatorname{Pr}\left(r / \eta_{7}\right) \times \operatorname{Pr}\left(\eta_{7}\right)+\operatorname{Pr}\left(r / \eta_{8}\right) \times \operatorname{Pr}\left(\eta_{8}\right)} \\
& =\frac{1 \times \frac{1}{2} q}{1 \times 0+1 \times \frac{1}{2} \times q+1 \times 0+1 \times \frac{1}{2}(1-q)}=q
\end{aligned}
$$

Similarly we obtain the conditional probabilities $\operatorname{Pr}\left(\eta_{5} / r\right)=0, \operatorname{Pr}\left(\eta_{7} / r\right)=0$ and $\operatorname{Pr}\left(\eta_{8} / r\right)=1-q$.

Given $q$ small, player $H$ will follow in his action the state that he observes almost surely, expecting that $D$ will realize this, and thus turn herself in the same direction. Essentially, $H$ is punished if he fails to report the signal that he has seen on the screen. The noise it contains, means that despite the truthful revelation, it is not always a correct reflection of the true state of nature and hence the expected payoffs reflect this by being less than 1 .

In terms of Figure 1, the prophesies which form a rational expectations equilibrium take the following form. $H$ prophesizes that when $D$ hears $l(r)$ she will believe that $H$ has observed $L^{\prime}\left(R^{\prime}\right)$ and therefore she will play $l^{\prime}\left(r^{\prime}\right)$ because she knows that if she takes the correct decision she will get an optimum payoff. $D$ prophezises that when $H$ sees $L^{\prime}\left(R^{\prime}\right)$, given that the noise is small, he will repeat the message sent by the machine and play $l(r)$ since if he transmits a correct signal he may get an optimum payoff, whereas if he transmits a signal that does not reflect the state of nature he will not irrespective of what $D$ plays.

We shall now discuss how these rational decisions of the agents are locked in a fixed point. As optimal reactions to each other's actions they confirm themselves.

The fixed point equilibrium is characterized as follows. $H$ plays from $I 1$ the choices $l$ and $r$ with probabilities $1-p_{1}$ and $p_{1}$ respectively, and from $I_{2}$ the choices $l$ and $r$ with probabilities $1-p_{2}$ and $p_{2}$ respectively. $D$ plays from $I_{3}$ the choices $l^{\prime}$ and $r^{\prime}$ with probabilities $1-z_{1}$ and $z_{1}$ respectively, and $I_{4}$ the choices $l^{\prime}$ and $r^{\prime}$ with probabilities $1-z_{2}$ and $z_{2}$ respectively.

If $D$ chooses $z_{1}=0$ and $z_{2}=1$, then the resulting expected payoff for $H$, ignoring $\frac{1}{2}$, is

$$
E_{1}=(1-q)\left(1-p_{1}\right)+q\left(1-p_{2}\right)+q p_{1}+(1-q) p_{2}
$$

which is maximized at $p_{1}=0$ and $p_{2}=1$. On the other hand for $p_{1}=0$ and $p_{2}=1$, we obtain

$$
E_{2}=(1-q)\left(1-z_{1}\right)+q\left(1-z_{2}\right)+q z_{1}+(1-q) z_{2}
$$

which, given that $q$ is small, is maximized at $z_{1}=0$ and $z_{2}=1$. Hence the the pairs $z_{1}=0, z_{2}=1$ and $p_{1}=0, p_{2}=1$ confirm each other as a fixed pair. Starting from one pair of rational decisions we go back and confirm it.

In terms of reaction functions the Kakutani fixed point theorem is seen as follows. Let $p=\left(p_{1}, p_{2}\right)$, $z=\left(z_{1}, z_{2}\right)$ be the two vectors and $R_{1}(z), R_{2}(p)$ the reaction functions (correspondences) of the helper and the driver respectively. The fixed point satisfies $(p, z) \in R_{1} \times R_{2}$.

We have analyzed the equilibrium $\left(l r, l^{\prime} r^{\prime}\right)$. Inserting back in our calculations the fraction $\frac{1}{2}$, the resulting payoffs are $E_{1}=1-q$ and $E_{2}=1-q$.

The pair $E_{1}=1-q$ and $E_{2}=1-q$ are the best possible payoffs and hence the best candidates for optimality because each time $H$ follows the message of the machine, i.e. he reports correctly, he is right with a high probability and $D$ follows his suggestion. So with a high probability, $1-q$, both players get each time payoff 1 . In expectation they get each time $\frac{1}{2}(1-q) \times 1$ and there are two such times. We discuss this further below. 
We can provide some further explanation with respect to the expected payoffs. A folded up tree can be obtained through backward induction. We are using the optimal strategies of $D$ given that $z_{1}=0, z_{2}=1$. In the folded up tree of Figure 1 it is clear the $H$ must use $l$ from point $I 1$ and $r$ from 2 .

Now, the PBE is a technical definition and as such it allows for other such equilibria as well. For example the following pairs of behavioural strategies $\left(r l, r^{\prime} l^{\prime}\right),\left(l l, l^{\prime} l^{\prime}\right)$, and $\left(r r, r^{\prime} r^{\prime}\right)$ form a PBE.

In Figure 2 we consider, in particular, the strategies $\left(r l, r^{\prime} l^{\prime}\right)$ which also form a PBE, but with payoff lower for $H$ than that corresponding to the pair $\left(l r, l^{\prime} r^{\prime}\right)$.

We shall now discuss also for the pair $\left(r l, r^{\prime} l^{\prime}\right)$ how these rational decisions of the agents form a fixed point and thus confirm themselves.

The fixed point equilibrium is found as follows. Again $H$ plays from $I_{1}$ the choices $l$ and $r$ with probabilities $1-p_{1}$ and $p_{1}$ respectively, and from $I_{2}$ the choices $l$ and $r$ with probabilities $1-p_{2}$ and $p_{2}$ respectively. $D$ plays from $I_{3}$ the choices $l^{\prime}$ and $r^{\prime}$ with probabilities $1-z_{1}$ and $z_{1}$ respectively, and $I_{4}$ the choices $l^{\prime}$ and $r^{\prime}$ with probabilities $1-z_{2}$ and $z_{2}$ respectively.

The mathematical analysis is supported by optimal decisions obtained by analyzing the graph. From Figure 2 we obtain the optimal decisions $p_{1}=1, p_{2}=0, z_{1}=1$ and $z_{2}=0$ If $D$ chooses $z_{1}=1$ and $z_{2}=0$, then the resulting expected payoff for $H$ is seen to be $E_{1}=0$ irrespective of $p_{1}$ and $p_{2}$. Therefore this value is also obtained $p_{1}=1, p_{2}=0$. On the other hand for $p_{1}=1, p_{2}=0$, we obtain, ignoring $\frac{1}{2}$,

$$
E_{2}=(1-q)\left(1-z_{2}\right)+q\left(1-z_{1}\right)+q z_{2}+(1-q) z_{1}
$$

which is maximized at $z_{1}=1$ and $z_{2}=0$. Hence the the pairs $z_{1}=1, z_{2}=0$ and $p_{1}=1, p_{2}=0$ confirm each other as a fixed pair. Starting from one pair of rational decisions we go back and confirm it.

We have analyzed the equilibrium $\left(r l, r^{\prime} l^{\prime}\right)$. Inserting back in our calculations the fraction $\frac{1}{2}$, the resulting payoffs are $E_{1}=0$ and $E_{2}=1-q$. In Figure 2 the beliefs of player $D$ are given for both the points in the information set $I_{3}$ and those in $I_{4}$. These are consistent with the moves up to these points and guarantee the optimality of the moves which follow.

We return now to the issue of the most likely equilibrium to prevail. From the four pure NE referred to above, it is easy to see that in the case of $\left(l l, l^{\prime} l^{\prime}\right)$ and $\left(r r, r^{\prime} r^{\prime}\right)$ the signal of $H$ has zero informational content. Hence the "updated" beliefs of $D$ in $I_{3}$ and $I_{4}$ are identical to the priors of the alternative states of nature. This leads to equilibrium expected payoffs of $E_{1}=E_{2}=\frac{1}{2}$. Regarding $\left(r l, r^{\prime} l^{\prime}\right)$, we see that the expected payoff for $H$ is zero. In other words, the sender will be punished for being untruthful in terms of revealing his private information regarding the state of nature as seen on the machine, as the probability that this is wrong is small. Hence if $H$ misreports what he has seen on the machine, then he is likely to have send an incorrect signal regarding the state of nature. He will be punished for this with a zero payoff. Given this, player $D$ can reasonably expect that $H$ has an interest to report truthfully what he has seen on the machine.

In cheap talk games speech serves the purpose of reinforcing a particular action and provides the evolutionary process rationale for choosing a particular equilibrium. Similarly, in a signaling game, $D$ will believe that the sender will send him true information unless he has a reason to deceive him. Using the idea of rational expectations it is obvious that $H$ has an interest to report the truth regarding his private information because failing to do so hurts him. Hence $D$ can reasonably expect and correctly guess that he will signal truthfully. This leads to the formation of a language (signaling) convention between the players.

Therefore, given $q$ small, the equilibrium $\left(I r, l^{\prime} r^{\prime}\right)$ in Figure 1 is the most likely to prevail, as it involves the maximum possible payoff for both $H$ and $D$ and hence is the best candidate for optimality in a repeated game of trial and error until a signaling convention is achieved.

Finally, we also consider a case of a pair of strategies which do not form a NE. As it is easy to see this is the case if in Figure 1 where all decisions are kept the same apart from changing for the driver $l^{\prime}$ to $r^{\prime}$ in $I_{3}$, i.e. the pair of strategies are $\left(l r, r^{\prime} r^{\prime}\right)$. In this case $D$ can change from $r^{\prime}$ in $I_{3}$ to $l^{\prime}$ and improve her payoff.

Case 2. $q$ is large. The optimal paths are shown in Figure 3 and Figure 4. It is easy to check that the heavy lines strategies form a NE. In Figure 3 only the strategies of $D$ are different. On the other hand, since the 
optimal actions of $H$ are the same for both small and large $q$ we shall be obtaining, through the Bayesian updating of conditional probabilities, the same beliefs for the nodes of $I_{3}$ and $I_{4}$ as in Figure 1. In the case of the equilibrium in Figure 3, $H$ does not take into account the fact that the machine is so faulty that it is more often wrong than right and he gets punished for truthfully sending what is likely to be an incorrect signal. Player $D$ does not believe the signal she received, turns in the correct direction and she is rewarded. As a result $E_{1}=0$ and $E_{2}=q$.

In Figure 4 we show an alternative NE for large $q$ and corresponding beliefs at the nodes of the information sets $I_{3}$ and $I_{4}$ and hence an alternative PBE to that in Figure 3. In this case both players make the correct move and they are both rewarded. Specifically, $H$ reports the opposite of what he sees, and both him and $D$ end up with payoffs $E_{1}=E_{2}=q>\frac{1}{2}$.

The pair $E_{1}=q$ and $E_{2}=q$ are the best possible payoffs and hence the best candidates for optimality. The machine is now very faulty and each time $H$ makes a guess he does not follow its message, i.e. he misreports, and he is right with a high probability and $D$ follows his suggestion. So with a high probability, q, both players get each time payoff 1 . In expectation they get each time $\frac{1}{2} q \times 1$ and there are two such times.

Again for completeness, we also consider a case of a pair of strategies which do not form a NE. This is the case, as it is easy to see, if in Figure 3 all decisions are kept the same apart from changing for the driver $l^{\prime}$ to $r^{\prime}$ in $I_{4}$.

Finally we note that it is easy to see that in both Figure 3 and Figure 4 the actions of the optimal paths satisfy Kakutani's fixed point theorem and form a rational expectation equilibrium.

Case 3. $q=\frac{1}{2}$. We can now have an equilibrium such that only information set $I_{3}$ or only $I_{4}$, as shown in Figure 5, is visited. Suppose that the latter is the case. With respect to the arbitrary beliefs for $I_{3}$ which is not visited by the optimal strategies, we can adopt beliefs such that the optimal choice is $r^{\prime}$.

Coming now to $I_{4}$, all its nodes are visited as player $H$ plays constantly $r$. The beliefs through Bayesian updating are as follows.

$$
\begin{aligned}
\operatorname{Pr}\left(\eta_{5} / r\right) & =\frac{\operatorname{Pr}\left(r / \eta_{5}\right) \times \operatorname{Pr}\left(\eta_{5}\right)}{\operatorname{Pr}\left(r / \eta_{5}\right) \times \operatorname{Pr}\left(\eta_{5}\right)+\operatorname{Pr}\left(r / \eta_{6}\right) \times \operatorname{Pr}\left(\eta_{6}\right)+\operatorname{Pr}\left(r / \eta_{7}\right) \times \operatorname{Pr}\left(\eta_{7}\right)+\operatorname{Pr}\left(r / \eta_{8}\right) \times \operatorname{Pr}\left(\eta_{8}\right)} \\
& =\frac{\frac{1}{2}(1-q)}{\frac{1}{2}(1-q)+\frac{1}{2} \times q+\frac{1}{2} \times q+\frac{1}{2}(1-q)}=\frac{1}{2}(1-q)
\end{aligned}
$$

Similarly we obtain the conditional probabilities $\operatorname{Pr}\left(\eta_{6} / r\right)=\frac{1}{2} q, \quad \operatorname{Pr}\left(\eta_{7} / r\right)=\frac{1}{2} q$ and $\operatorname{Pr}\left(\eta_{8} / r\right)=\frac{1}{2}(1-q)$.

We now have that $D$ plays $r^{\prime}$ from $I_{3}$ and $l^{\prime}$ from $I_{4}$, while $H$ always plays $r$. We want to check that $\left(r r, r^{\prime} l^{\prime}\right)$ is a NE. Given the strategies of one player we have to consider the alternative actions of the other. Given constant $r$, player $D$ has made an optimal choice. For the response of $H$ to $r^{\prime} l^{\prime}$ of $D$, it is straightforward, inspecting the graph, that $H$ cannot change a strategy and improve his payoff. Hence $\left(r r, r^{\prime} l^{\prime}\right)$ forms a NE. The payoffs are $E_{1}=0$ and $E_{2}=\frac{1}{2}$. Player $H$ is punished if D decides to play $l^{\prime}$ in $I_{4}$.

We shall now discuss how these rational decisions of the agents are locked in a fixed point. As optimal reactions to each other's actions they confirm themselves and the form a rational expectations equilibrium.

Suppose we consider $z_{1}=1$ and $z_{2}=0$. These values mean that $E_{1}=0$ irrespective of $p_{1}$ and $p_{2}$, and therefore we can choose $p_{1}=1$ and $p_{2}=1$ which $D$ takes as given and calculates, ignoring $\frac{1}{2}$, 


$$
E_{2}=(1-q)\left(1-z_{2}\right)+q\left(1-z_{2}\right)+q z_{2}+(1-q) z_{2}
$$

which means that $E_{2}=\frac{1}{2}$ irrespective of $z_{1}$ and $z_{2}$ and therefore we can choose $z_{1}=1$, and $z_{2}=0$.

In fact, we have a multiplicity of equilibria as D could play instead $r^{\prime} r^{\prime}$. The pairs of strategies $\left(l l, l^{\prime} l^{\prime}\right)$ and $\left(r r, r^{\prime} r^{\prime}\right)$ form also equilibria. In each of these case the beliefs at the nodes of the information sets of $D$ are properly adjusted, but the payoffs of $H$ and $D$ are the same. That is $E_{1}=\frac{1}{2}$ and $E_{2}=\frac{1}{2}$. A general argument can be advanced that the pair $E_{1}=E_{2}=\frac{1}{2}$ are the best possible payoffs and hence the best candidates for equilibrium.

The machine is now faulty with probability $\frac{1}{2}$. Each time $H$ makes a guess he has an equal chance to report or misreport the correct state of nature and when $D$ responds she has an equal chance of discovering the true state of nature.

Now, with respect to $D$, since she is rewarded for guessing correctly, she will always get expected payoff $E_{2}=\frac{1}{2}$. However the reward of $H$ is calculated, as explained above, in a more complicated manner. He will only get a payoff of 1 if his signal coincides with the correct choice of $D$; otherwise he gets $E_{1}=0$. It is therefore possible that $H$ gets payoff 0 because he has signaled the opposite of the action taken by $D$. On the other hand the latter by making a constant choice secures for himself payoff $E_{2}=\frac{1}{2}$.

Finally, we state a pair of strategies which do not form a NE. This is the case, as it is straightforward to see, of the pair $\left(r r, l^{\prime} l^{\prime}\right)$. If $D$ chooses $l^{\prime} l^{\prime}$ then $H$ can improve his payoff by switching to $l l$.

\section{Further Discussion and Conclusions}

We have analysed a sequential game concerning instructions and decisions to and by players, where nature selects a state and a machine transmits a noisy signal which is received by a helper, $H$. The latter decides whether to truthfully report this signal to a driver, $D$. For a small noise the equilibrium outcome, not the payoffs, is the same as in the case $q=0$ discussed in the earlier paper, but for a large noise $H$ will be punished for truthfully sending to $D$ a false message.

With respect to payoffs, both players know that if the noise in the signal is small, announcements by $H$ which are not reflective of the state of nature will lead to outcomes that will hurt them. The incentives of $H$ and $D$ are compatible in the sense that $D$ can reasonably expect and correctly guesses that $H$ has an incentive to truthfully report a signal, when that signal is likely to be a correct reflection of the state of nature.

In the case of a noisy signal $H$ may receive an incorrect signal from the machine which he reports truthfully. Even if the final choice is correct, his expected payoff will in this case be zero. So truthfulness no longer automatically implies that a correct signal is sent.

As argued in [2], the assumption of an aligned interest between the helper, as the sender of a signal, and the driver as the receiver is found in much of the existing literature. The incentive of $H$ to choose $l r$ rather than $r l$ no longer applies if $H$ 's payoff is no longer dependent on being correct. This would make the helper careless in terms of his reporting. He knows that his payoff is dependent on the final outcome which is determined by the actions of the driver irrespective of whether his signal reflects the true state of nature. The result of this assumption is that the formation of a convention is made less likely. This will still hold true in the case of a noisy signal, as long as $q<\frac{1}{2}$.

In contrast, in our model, $H$ knows that the probability of his signal to $D$ correctly reflecting the state of nature is more likely if he truthfully reports to $D$ the signal he has received from the machine. Since being correct is a necessary condition for his getting a strictly positive payoff, the case is identical to the one discussed in [2] where $q=0$.

In the case of a large $q$, the incentive of $H$ to report a signal that correctly reflects the state of nature still 
applies. But in this case, it means that $H$ has an incentive to report the opposite of what has been transmitted to him by the machine. In other words, it is not in the interests of $H$ to truthfully report the message he has received by the machine (private information). By reporting exactly the opposite of what he sees, $H$ is more often correct than not. Hence in this case there is an inverse relationship between correctness and truthfulness.

In both Case 1 and Case 2 we get a number of different PBE. Only one of these seems to be justified in providing an equilibrium which introduces a self enforcing behaviour and also provides an optimum outcome for both players.

In Case 3 there is also a number of equilibria. However from these only two PBE secure an optimum payoff for both players. These are the ones where both $H$ and $D$ make constant choices from both of their corresponding information sets. However unlike the PBE in Cases 1 and 2, these optimal payoffs do not exceed the default payoff of $D$ choosing an action randomly (i.e. $\frac{1}{2}$ ) as the informational value of the signals by $H$ is zero.

\section{Acknowledgements}

We wish to thank Professor Dimitrios Tsomokos of Oxford University for his suggestions and penetrating comments which led to a substantial improvement of the final draft. Of course responsibility for all short comings stays with the authors.

\section{References}

[1] Lewis, D. (1969) Convention. Harvard University Press, Cambridge, MA.

[2] Dassiou, X. and Glycopantis, D. (2013) A Tree Formulation for Signaling Games. Game Theory, 2013, Article ID: 754398, 12p.

[3] Blume, A. (2012) A Class of Strategy-Correlated Equilibria in Sender-Receiver Games. Games and Economic Behavior, 75, 510-517. http://dx.doi.org/10.1016/j.geb.2012.03.008

[4] Rubinstein, A. (2000) Economics and Language. Cambridge University Press, Cambridge, UK. http://dx.doi.org/10.1017/CBO9780511492358

[5] Cho, I. and Kreps, D.M. (1987) Signaling Games and Stable Equilibria. The Quarterly Journal of Economics, 102, 179-221. http://dx.doi.org/10.2307/1885060

[6] Pawlowitsch, C. (2008) Why Evolution Does Not Always Lead to an Optimal Signaling System. Games and Economic Behavior, 63, 203-226. http://dx.doi.org/10.1016/j.geb.2007.08.009

[7] Huttegger, S.M. (2007) Evolutionary Explanations of Indicatives and Imperatives. Erkenntnis, 66, 409-436. http://dx.doi.org/10.1007/s10670-006-9022-1

[8] Skyrms, B. (2010) The Flow of Information in Signaling Games. Philosophical Studies, 147, 155-165. http://dx.doi.org/10.1007/s11098-009-9452-0

[9] Stokke, A. (2014) Truth and Context Change. Journal of Philosophical Logic, 43, 33-51. http://dx.doi.org/10.1007/s10992-012-9250-6

[10] Glycopantis, D., Muir, A. and Yannelis, N. (2003) On Extensive form Implementation of Contracts in Differential Information Economies. Economic Theory, 21, 495-526. (Reprinted in Aliprantis, C.D., Arrow, K.J., et al., Eds., Assets, Beliefs, and Equilibria in Economic Dynamics, Springer-Verlag, 2004, 323-354.) 
Scientific Research Publishing (SCIRP) is one of the largest Open Access journal publishers. It is currently publishing more than 200 open access, online, peer-reviewed journals covering a wide range of academic disciplines. SCIRP serves the worldwide academic communities and contributes to the progress and application of science with its publication.

Other selected journals from SCIRP are listed as below. Submit your manuscript to us via either submit@scirp.org or Online Submission Portal.
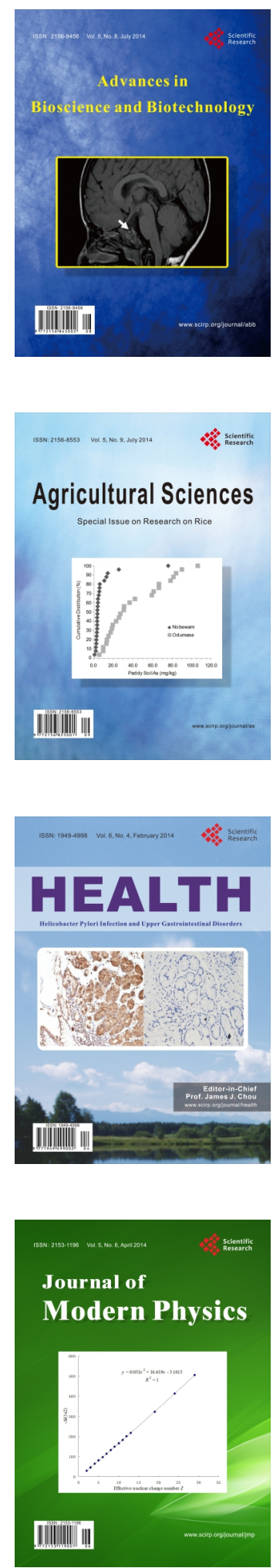
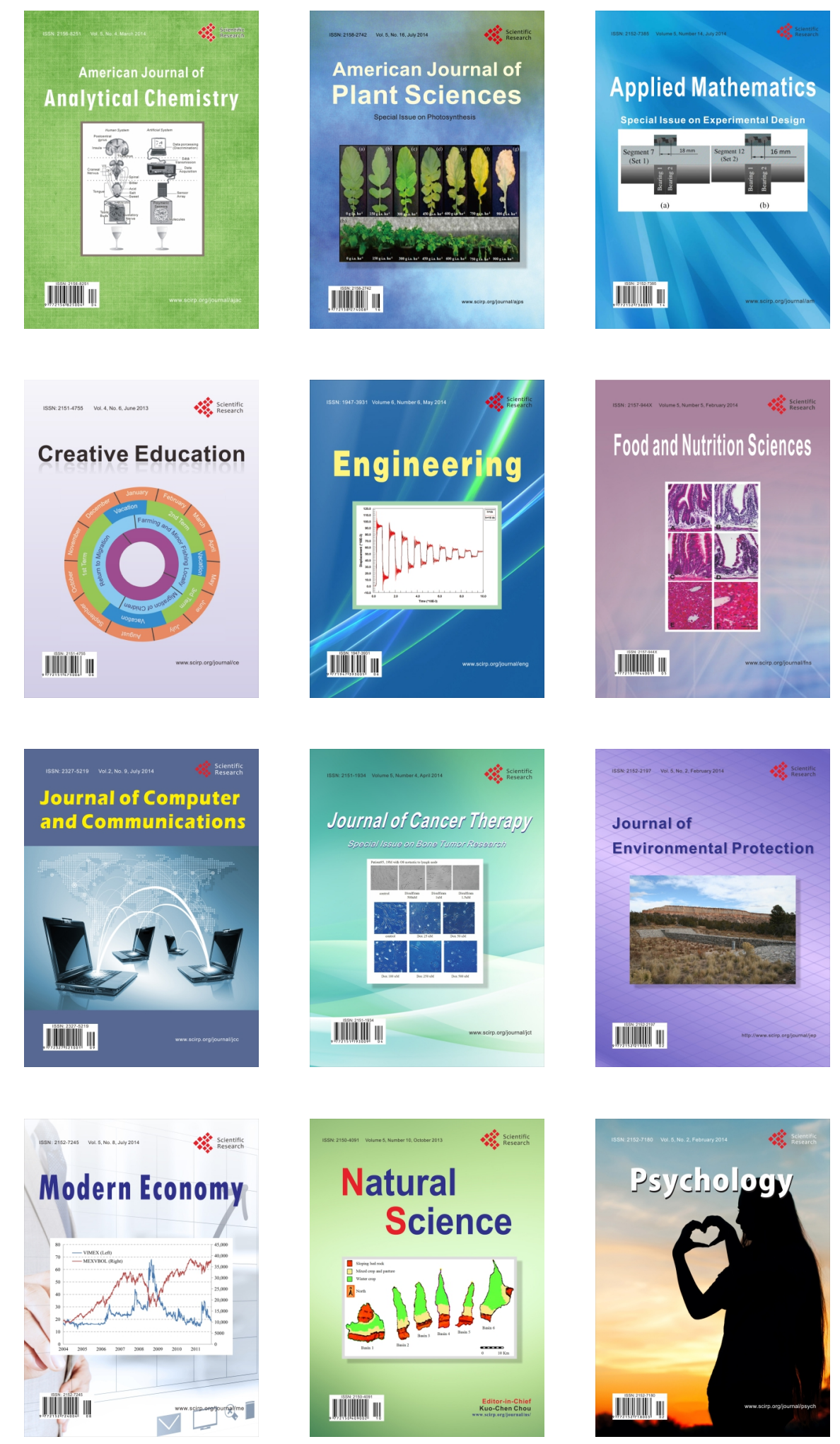